\title{
Influence of Roughness on Contact Force Estimation During Active Touch
}

\author{
Kaho Shirakawa $^{(\otimes)}$ and Yoshihiro Tanaka ${ }^{(凶)}$ \\ Nagoya Institute of Technology, Gokiso-cho, Showa-ku, Nagoya 466-8555, Japan \\ k.shirakawa.680@nitech.jp, tanaka.yoshihiro@nitech.ac.jp
}

\begin{abstract}
Haptic sensations consist of cutaneous information elicited on the skin and kinesthetic information collected by the musculoskeletal system; there is a bidirectional relationship between haptic sensations and exploratory movements. Previous researches have investigated exploratory movement strategies for active haptic perception and the influence of exploratory movements on haptic sensations. This paper investigates the influence of roughness on the estimation of contact force during the active touch of samples with different textures. Two stimuli with different roughness were prepared and the contact force was measured when the participants rubbed pairs of samples with identical and different stimuli under the instruction of keeping the contact force constant. Eleven healthy adults participated in the experiment. The results showed that the accuracy of controlling the contact force for identical samples was not significantly different between coarse and smooth textures, whereas the contact forces between the coarse and the smooth sample when rubbing pairs of them were significantly different for six of eleven participants. These participants overestimated the contact force exerted for the coarse stimulus in comparison with the smooth stimulus. Thus, the results imply that textures during rubbing can yield perceptual bias for the contact force exerted; however, there are individual differences for this effect. There might be a complex perception mechanism for kinesthetic information involving cutaneous information.
\end{abstract}

Keywords: Perceptual bias $\cdot$ Roughness $\cdot$ Contact force

\section{Introduction}

Haptic sensations consist of cutaneous information elicited on the skin and kinesthetic information collected by the musculoskeletal system [1]. Some types of cutaneous information are pressure, vibration, and temperature. Exploratory movements, which are used to collect kinesthetic information, affect the mechanical phenomenon related to the collection of cutaneous information. For example, Lederman and Talyor [2] demonstrated that roughness perception enhances with

This work was supported by JSPS KAKENHI Grant Number JP19K22871.

(C) The Author(s) 2020

I. Nisky et al. (Eds.): EuroHaptics 2020, LNCS 12272, pp. 140-148, 2020.

https://doi.org/10.1007/978-3-030-58147-3_16 
the rise of the contact force. Natume et al. [3] reported that temporal vibrotactile information, spatial pressure distribution, and friction information affect subjective roughness ratings, indicating that the cognitive loads to each parameter differ among individuals. Thus, haptic perception is complex, and relevant factors are related to each other, including individual differences in cognitive processing. Furthermore, at high-level cognition, other modalities also influence haptic perception as observed in the size-weight illusion [4].

In active touch, there is sensory-motor control; this implies a bidirectional relationship between haptic sensations and exploratory movements. We consciously and unconsciously modify exploratory movements (e.g. contact force and scanning velocity) according to haptic sensations such as hardness, texture, and temperature. Previous studies have investigated the relationship between exploratory movements and haptic perception: Lezkan et al. [5] showed that sensory signals led to lower forces for more compliant objects; Tanaka et al. [6] showed that the variance of the contact force was larger for smooth stimuli than for coarse stimuli in a discrimination task; and Smith et al. [7] demonstrated that participants used a larger contact force for detecting a small concave object than for a small convex object. Thus, many previous works have been focused on exploratory movement strategies for active haptic perception, and as can be seen in [2], the influence of exploratory movements on haptic sensations has been also investigated. However, the influence of haptic sensations on the kinesthetic sensation involved in exploratory movements has been hardly investigated. Exploratory movements like contact force have been often controlled or measured in haptics researches. However, the kinesthetic sensations involved in exploratory movements may be affected by haptic sensations. In other aspect, previous researches on motor control have showed that perception of force and weight is affected by muscle activities [8].

This paper investigates the influence of roughness on the estimation of the contact force during active touch for different textures. Our hypothesis is that roughness promotes perceptual bias for the contact force exerted when rubbing samples of different textures with the fingertip. Two stimuli with different roughness were prepared and the contact force was measured when pairs of the same stimuli and different stimuli were rubbed under the instruction of keeping the same contact force. Differences in the contact force between the pairs were calculated and investigated.

\section{Materials and Methods}

\subsection{Participants}

Eleven healthy adults (five males and six females, aged 19-32) participated in the experiment. According to Coren's dominant hand discrimination test [9], the participants were strongly right-handed. The participants were naive about the purposes of the experiment and gave their written informed consent before participating in the experiment; they were instructed to use their dominant index 
finger during the tests. The experiment was approved by the Ethics Committee of Nagoya Institute of Technology.

\subsection{Stimuli and Experimental Setup}

Two types of stimuli, a coarse sample (C) and a smooth sample (S), were used in this experiment as shown in Fig. 1. Glass beads (Toshin Riko; glass beads no. 005 and no. 1) were attached to a flat $100 \times 60 \mathrm{~mm}$ acrylic plate with doublesided tape. The particle diameter of the glass bead was approximately $1.0 \mathrm{~mm}$ $(0.991-1.397 \mathrm{~mm})$ and $0.05 \mathrm{~mm}(0.037-0.063 \mathrm{~mm})$ for sample $\mathrm{C}$ and sample $\mathrm{S}$, respectively. The participants could distinguish between the roughness of these stimuli according to the results of a previous study [3]. The experiment was performed using pairs consisting of $\mathrm{C}$ and $\mathrm{C}, \mathrm{S}$ and $\mathrm{S}$, and $\mathrm{S}$ and $\mathrm{C}$. Thus, two identical samples for each stimulus type were prepared for a total of 4 samples and were used in random order for the experiment.

The experimental setup is shown in Fig. 2. Two samples were placed on a six-axis force sensor (ATI, Gamma) that was used to measure the contact force exerted during rubbing the samples. A laptop computer and a data acquisition (DAQ) module (National Instruments, NI USB-6218) were used to collect the contact force data. The sampling frequency was $1 \mathrm{kHz}$, and a low-pass filter with a cutoff frequency of $10 \mathrm{~Hz}$ were used for smoothing the data collected.

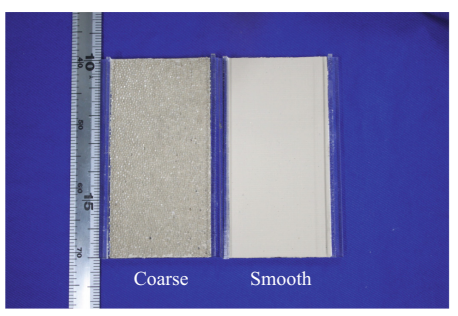

Fig. 1. Samples used in the experiment.

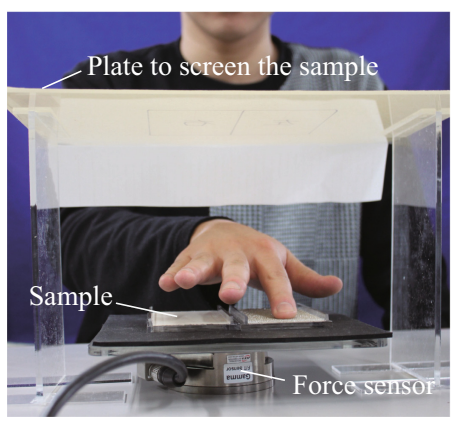

Fig. 2. Experimental setup.

\subsection{Procedure}

As shown in Fig. 2, two samples were arranged side by side in front of the participant. The participants were instructed to rub each sample from the back edge to the front edge. They were also instructed to rub each sample alternately, for a total of three times per sample (six strokes in total for one trial). On half of the trials, the participants firstly rub the sample placed on the left side, and on the other half of the trials, firstly rub the sample placed on the right side. The sample order was randomized within all trials for each participant. Regarding 
contact force, the participants were instructed to rub the two samples, exerting a constant contact force during each trial. In a preliminary test, when the exerted contact force was extremely small, it seemed that the participants relaxed and only used the weight of their own fingers and arms to exert the force. This case resembles feedforward control and implies that the participants might have not used finger motor control. Thus, the participants were allowed to use their preferred contact force, but the experimenter instructed them to slightly increase it when the exerted force was less than $0.3 \mathrm{~N}$. The rubbing distance was approximately $100 \mathrm{~mm}$, and the rubbing velocity was kept constant at approximately $100 \mathrm{~mm} / \mathrm{s}$ by instructing the participants to listen to a metronome sound of 60 beats per minute by means of a headphone. As shown in Fig. 2, the participants could not see the samples or hear any sound produced by rubbing the sample. For these purposes, a blind plate mounted on the experimental setup and headphones were employed, respectively.

Three pairs of stimuli (C-C, S-S, and S-C) were used in the experiment. The experiment consisted of three sessions; in each session, three pairs were presented twelve times (four times each pair) in random order, considering the position (left or right) for starting the assessment and two samples for each stimulus. In total, 36 trials were conducted for each participant.

\subsection{Data Analysis}

Figure 3 shows an example of contact force data collected for one trial. During each trial, the two samples were rubbed alternatively three times (1st, 3rd, and 5 th stroke for the firstly-rubbed sample, and 2nd, 4th, and 6th stoke for the secondly-rubbed sample). The force sensor output signals were extracted from the collected data. The rubbing period corresponded to $0.5 \mathrm{~s}$ within one stroke, from $0.25 \mathrm{~s}$ before to $0.25 \mathrm{~s}$ after the center point between the start and end points of the rubbing process. Approximately $50 \%$ of the whole rubbing period was used for the analysis. The start and end points of the rubbing process were detected using a $0.2 \mathrm{~N}$ contact force threshold for each stroke. Only for 3 trials out of the 396 trials, a threshold of $0.5 \mathrm{~N}$ was used because the participants kept touching the base plate of the force sensor where the two samples were placed to find the starting position.

The mean contact force was calculated from the extracted data of 3 strokes for each sample and then the difference between the mean contact force of the two samples presented (defined as $\alpha$ ) was calculated for each trial. For the S-C pairs, the difference was calculated by subtracting the contact force of sample $\mathrm{C}$ from the contact force of sample S. Twelve $\alpha$ values were obtained for each S-C, C-C, and S-S pairs presented to each participant. Additionally, the contact force and the sensitivity were compared by using the data for S-S and C-C. The mean contact force $F_{z}$ within one trial (6 strokes) and the quotient $|\alpha| / F_{z}$ by using the absolute difference $|\alpha|$ and $F_{z}$ were calculated for the pairs consisting of identical samples (S-S and C-C). For the pairs S-S and C-C, the samples presented the same stimulus; therefore, $|\alpha| / F_{z}$ represents the sensitivity to keep the contact force constant. 


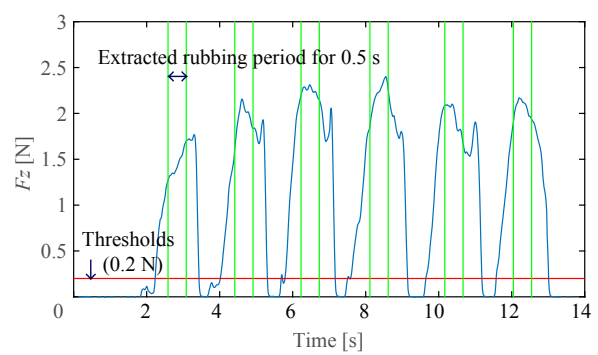

Fig. 3. Example of contact force for one trial. Two samples were rubbed alternatively for a total of 3 stroke for each. The data for the analysis was extracted for each stroke by using a threshold.

Considering individual differences, this study conducted statistical analysis for each participant. A Wilcoxon rank sum test with Bonferroni correction was performed for each participant in order to compare the difference in contact force $\alpha$ to zero, for each S-C, C-C, and S-S. The contact force $F_{z}$ and the sensitivity $|\alpha| / F_{z}$ were compared between two conditions of identical samples (C-C and S-S) with a Wilcoxon rank sum test with Bonferroni correction for each participant. The significance level was set to 0.05 .

\section{Results}

\subsection{Differences in Contact Force}

Figure 4 shows the difference in contact force $\alpha$ of the three conditions (S-C, C-C, and S-S) for each participant. It can be seen that the tendency of $\alpha$ was different among participants. The Wilcoxon rank sum test with Bonferroni correction was performed for each participant in order to compare $\alpha$ to zero. Regarding S-C, the statistical analysis showed that six participants presented significant positive values of $\alpha(p<0.05$ for 3 participants and $p<0.01$ for 3 other participants), whereas, for the other participants, there were no significant differences. As for C-C and S-S, no significant differences were observed.

\subsection{Contact Force and Sensitivity for Pairs of Identical Samples}

Figure 5 shows the mean contact force $F_{z}$ for the pairs consisting of identical samples (C-C and S-S) for each participant. The mean contact force and standard deviation for all participants were $1.24 \pm 0.57 \mathrm{~N}$ for C-C and $1.42 \pm 0.56 \mathrm{~N}$ for SS. The Wilcoxon rank sum test with Bonferroni correction for each participant showed that there was no significant difference in sensitivity between the two conditions (C-C and S-S). The contact force for all trials and all participants was within $0.31-3.7 \mathrm{~N}$. This range is within that reported in previous studies on texture rubbing [10]. The contact force for $\mathrm{S}-\mathrm{C}$ was within the range of that for C-C and S-S. 

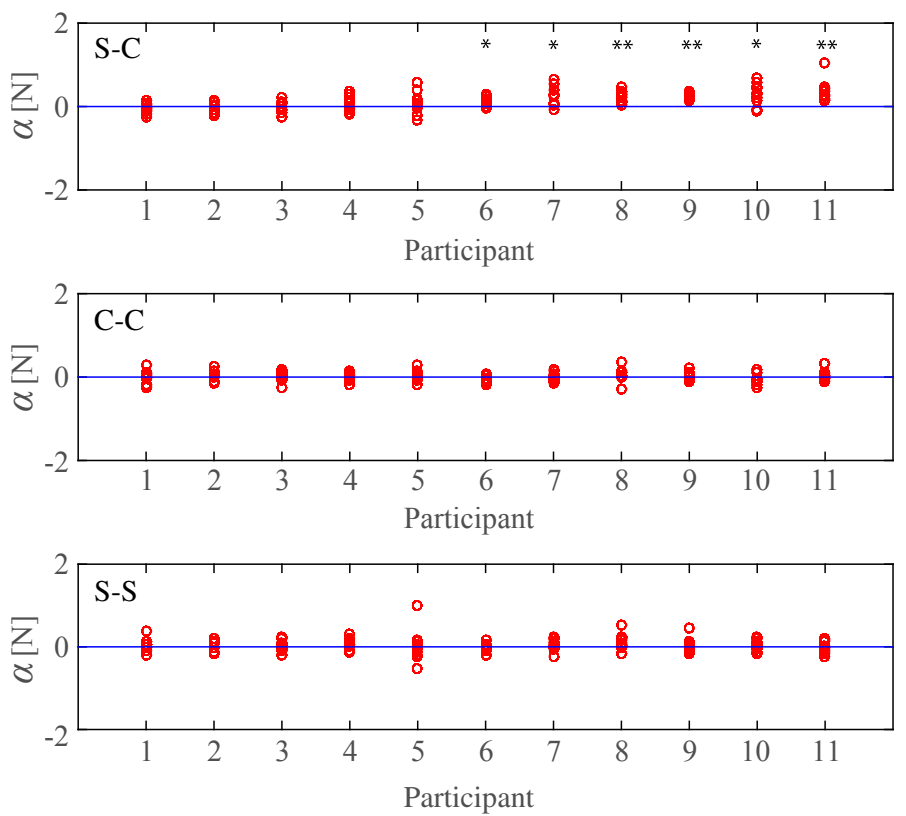

Fig. 4. Contact force differences of conditions S-C, C-C, and S-S for each participant. The results of all trials were plotted for all participants ordered by size of the mean $\alpha$; * and ${ }^{* *}$ denote $p<0.05$ and $p<0.01$, respectively.

Figure 6 shows the mean sensitivity $|\alpha| / F_{z}$ for the pair of coarse samples (C-C) and smooth samples (S-S) for each participant. The mean sensitivity and standard deviation were $0.08 \pm 0.02$ for $\mathrm{C}-\mathrm{C}$ and $0.09 \pm 0.03$ for S-S. The Wilcoxon rank sum test with Bonferroni correction for each participant showed that there was no significant difference in sensitivity between the two conditions (C-C and S-S).

\section{Discussion}

The mean contact force results shown in Fig. 5 indicate that the contact force exerted was not significantly different between pairs of coarse samples and pairs of smooth samples, despite of being allowed to use a preferred contact force during each trial. A previous study with sandpapers demonstrated that contact force was smaller for rough samples than for smooth samples and discussed a possible reason of the discomfort [6]. In our experiment, samples were not discomfort due to using glass beads. Thus, no significant difference in the contact force was observed. For some participants, it appears that data point in Fig. 5 is distributed in two groups because they trended to use different force among each session. 


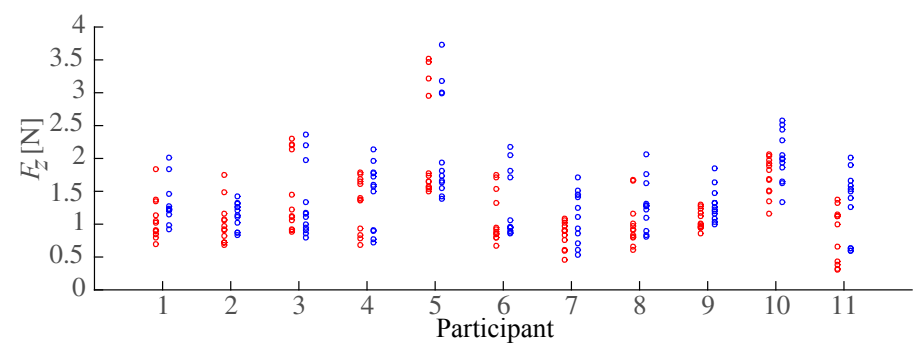

Fig. 5. Contact force $F_{z}$ of conditions C-C and S-S for each participant. The results of all trials were plotted for all participants ordered by size of the mean $\alpha$ (see Fig. 4); Red dots and blue dots are C-C and S-S, respectively.

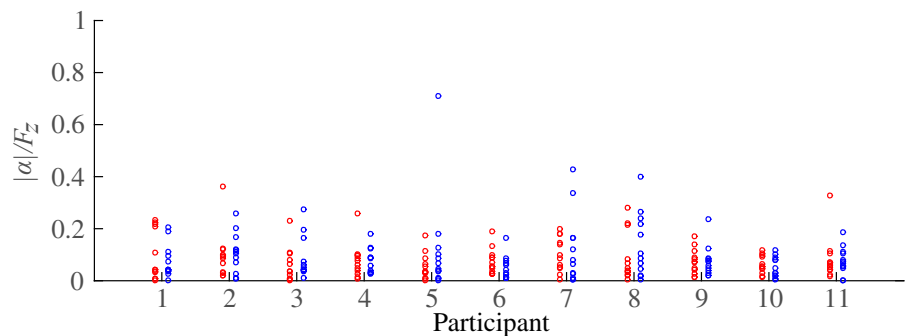

Fig. 6. Sensitivity of conditions C-C and S-S for each participant. The results of all trials were plotted for all participants ordered by size of the mean $\alpha$ (see Fig. 4); Red dots and blue dots are C-C and S-S, respectively. (Color figure online)

The sensitivity results shown in Fig. 6 indicate that the accuracy of the contact force exerted on the two samples presented was not significantly different between pairs of coarse samples and pairs of smooth samples. The result indicated that the participants could keep the contact force constant within an error of approximately $10 \%$ when rubbing identical samples for both coarse and smooth stimuli. Vibrotactile stimulation can promote a masking effect on haptic/tactile perception [11]; however, the experimental results indicated that the accuracy of the contact force did not become significantly low even for coarse samples, which elicited larger vibrotactile stimulation as compared with the smooth samples.

The results on contact force difference shown in Fig. 4 demonstrated that 6 out of 11 participants exerted a significantly larger contact force on the smooth sample than on the coarse sample under S-C condition, whereas the others did not exert a significantly different contact force. Moreover, none of the participants exerted a significantly different contact force under S-S and C-C conditions. The results shown in Fig. 5 indicated that the comfort did not significantly influence the contact force exerted for any participant in this experiment, and the results shown in Fig. 6 indicated that the sensitivity did not become significantly low for the coarse stimulus. Therefore, the significant differences observed 
in Fig. 4 imply the presence of perceptual bias for contact force; these participants overestimated the contact force exerted for the coarse stimulus. This indicates that, for some individuals, the contact force sensation based on kinesthetic perception can be affected by the roughness sensation based on cutaneous perception; there might be an integration mechanism for kinesthetic and cutaneous information regarding contact force perception. Additionally, it seems that the cognitive loads related to them differ among individuals; the results showed no significant differences for 5 out of 11 participants, whereas there were individual differences among the participants that presented significant differences, as shown in Fig. 4.

Here, as this experiment used only two different roughness stimuli, the discussion has limitations. A more diverse set of textures should be investigated to determine the physical factor that derives the perceptual bias of contact force. Furthermore, perceptual bias on exploratory movements involving the scanning velocity will be investigated in future work.

\section{Conclusion}

The present paper investigated contact force exerted during rubbing different samples with coarse and smooth textures. For pairs of different textures, six out of eleven participants significantly overestimated the contact force to the coarse textures whereas the others did not use significantly difference force. For identical samples of coarse or smooth textures, none of the participants used significantly different contact force. Previous studies have showed that cutaneous perceptions, like those involved in roughness rating, are affected by kinesthetic information [2]. Herein, our results implied that kinesthetic perceptions like contact force can be also affected by cutaneous information. There might be a complex perception mechanism for kinesthetic information involving cutaneous information. Our findings might be useful for the evaluation of haptic sensations and the development of haptic devices. In future work, a more diverse set of textures should be investigated to determine the driving factor of the perceptual bias of the contact force. We will also investigate the influence of other cutaneous information like friction and temperature on contact force perception, involving the scanning velocity, and the possible causes of the differences in cognitive loading between participants.

\section{References}

1. Loomis, J.M., Lederman, S.J.: Tactual perception. In: Boff, K.R., Kaufman, L., Thomas, J.P. (eds.) Handbook of Perception and Human Performance, Cognitive Processes and Performance. Wiley-Interscience, Hoboken (1986)

2. Lederman, S.J., Taylor, M.M.: Fingertip force, surface geometry, and the perception of roughness by active touch. Percept. Psychophys. 12(5), 401-408 (1972). https://doi.org/10.3758/BF03205850 
3. Natume, M., Tanaka, Y., Kappers, A.M.L.: Individual differences in cognitive proceeding for roughness rating of fine and coarse textures. PLoS One 14(1), e0211407 (2019)

4. Flanagan, J.R., Beltzner, M.A.: Independence of perceptual and sensorimotor predictions in the size-weight illusion. Nat. Neurosci. 3(7), 737-741 (2000)

5. Lezkan, A., Drewing., K.: Predictive and sensory signals systematically lower peak forces in the exploration of softer objects. In: Proceedings of 2015 IEEE World Haptics Conference, pp. 69-74 (2015)

6. Tanaka, Y., Bergmann Tiest, W.M., Kappers, A.M., Sano, A.: Contact force and scanning velocity during active roughness perception. PLoS One 9(3), e93363 (2014)

7. Smith, A.M., Gosselin, G., Houde, B.: Deployment of fingertip forces in tactile exploration. Exp. Brain Res. 147(2), 209-218 (2002). https://doi.org/10.1007/ s00221-002-1240-4

8. Jones, L.A.: Perception of force and weight: theory and research. Psychol. Bull. J. 100(1), 29-42 (1986)

9. Coren, S.: The Left-Hander Syndrome. Vintage Books, New York (1993)

10. Natsume, M., Tanaka, Y., Bergmann Tiest, W.M., Kappers, A.M.L.: Skin vibration and contact force in active perception for roughness ratings. In: 2017 Proceedings of IEEE RO-MAN, pp. 1479-1484 (2017)

11. Gescheider, G.A., Verrillo, R.T., Van Doren, C.L.: Prediction of vibrotactile masking functions. J. Acoust. Soc. Am. 72, 1421-1426 (1982)

Open Access This chapter is licensed under the terms of the Creative Commons Attribution 4.0 International License (http://creativecommons.org/licenses/by/4.0/), which permits use, sharing, adaptation, distribution and reproduction in any medium or format, as long as you give appropriate credit to the original author(s) and the source, provide a link to the Creative Commons license and indicate if changes were made.

The images or other third party material in this chapter are included in the chapter's Creative Commons license, unless indicated otherwise in a credit line to the material. If material is not included in the chapter's Creative Commons license and your intended use is not permitted by statutory regulation or exceeds the permitted use, you will need to obtain permission directly from the copyright holder.

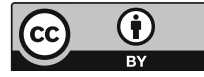

